ISSN (O) 2589-8779 | (P) 2589-8760

CMRO 04 (05), 940-949 (2021)

RESEARCH ARTICLE

\title{
Convalescent Plasma to Limit Coronavirus Associated Complications: A Proof of Concept Phase-2 Clinical Study at a designated COVID-19 hospital in Mumbai city
}

\author{
B. Pardiwalla ${ }^{1}$ A. Yadav² $\mid$ A. Bhatia ${ }^{2} \mid$ K. Toraskar ${ }^{1} \mid$ V. Sharma ${ }^{1} \mid$ M. Shah ${ }^{*} \mid$ R. Gutte \\ D. Kumbhar ${ }^{2}$ | D. Gohel ${ }^{2}$ | P. Narayan ${ }^{2}$ | H. Savla ${ }^{1}$ | N. Nagda' ${ }^{1}$ A. Bopardikar ${ }^{3}$
}

${ }^{1}$ Wockhardt's Hospital, near Police Station, 1877, Doctor Anandrao Nair Marg, Near Agripada, Mumbai Central, Mumbai, Maharashtra, India - 400011

${ }^{2}$ Wockhardt Ltd., Wockhardt Towers, Bandra Kurla Complex, G Block BKC, Bandra Kurla Complex, Bandra East, Mumbai, Maharashtra, India - 400051

${ }^{3}$ Mahatma Gandhi Hospital Blood Bank, 2nd Floor, Mahatma Gandhi Sevamandir, Swami Vivekananda road, opposite Bandra Lake, Bandra West, Mumbai, Maharashtra, India 400050

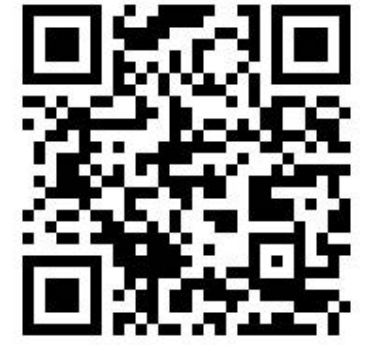

\begin{abstract}
Objective: With few treatment options available to manage coronavirus disease 2019 (COVID-19), health systems devised strategies to manage covid-19 using repurposed drugs and revisiting older strategies, such as convalescent plasma. This study was planned to evaluate safety and efficacy of Anti-SARS-CoV-2 convalescent plasma in hospitalized subjects with COVID-19.
\end{abstract}

Method: An open label, single centre, two arm, prospective, randomised, controlled exploratory phase 2 study was conducted at a covid-19 designated center. 20 subjects ( $\geq 18$ years) were admitted to hospital (screened 15 June to 27 July 2020) with confirmed moderate covid-19 (partial pressure of oxygen in arterial blood/ fraction of inspired oxygen $(\mathrm{PaO} 2 / \mathrm{FiO} 2)$ ratio between $200 \mathrm{~mm} \mathrm{Hg}$ and $300 \mathrm{~mm} \mathrm{Hg}$ or a respiratory rate of more than 24/ min with oxygen saturation $93 \%$ or less on room air): 10 subjects were assigned to convalescent plasma with standard treatment (test arm) and 10 subjects to standard treatment only (control arm). Subjects in the test arm received either single or two doses of convalescent plasma 24 hours apart based on their clinical condition as per investigator's discretion. Results: Subjects in test arm showed earlier resolution of symptoms of fever, shortness of breath and cough and the mean duration for RT-PCR test turning negative was better in the test arm. One subject in the control arm progressed to severe ARDS, while none in test arm progressed to severe ARDS. There was no difference in the use of respiratory support (invasive and non-invasive ventilation) between the 2 arms. There was no mortality observed in the study and no serious adverse reaction observed with the transfusion of convalescent plasma in the study.

Conclusion: This was an exploratory proof of concept study to explore the effectiveness of convalescent plasma in COVID-19 subjects and sample size was not large enough to detect a statistically significant difference however subjects in test arm of this study showed better outcomes in few of the efficacy parameters as compared to subjects in control arm. The use of convalescent plasma transfusion was also observed to be safe.

Keywords: COVID-19, Convalescent plasma, ARDS, RT-PCR, AntiSARS-CoV-2, standard treatment. 


\section{1 | INTRODUCTION}

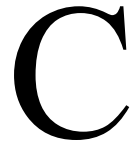

oronavirus or novel coronavirus which is taxonomically termed as SARS-CoV-2 and named by World Health organization (WHO) as COVID-19 which emerged from Wuhan city, Hubei

Province of China by the end of 2019 has caused unprecedented panic across the world. The rapid transmission of this virus from human to human made the World Health Organization (WHO) to declare this as the public health emergency of international concern and called it as global pandemic (1).

The first case of the COVID-19 pandemic in India was reported on January 30, 2020. As on April 25, 2021, the Ministry of Health and Family Welfare, Government of India has reported 17.6 million cases and 14.6 million recovered cases with $1,98,000$ deaths (2). India is currently grappling with the second wave of covid-19 infection with much more number of average daily cases and deaths as compared to last year.

Passive antibody therapy involves the administration of antibodies to a given agent to a susceptible individual for the purpose of preventing or treating an infectious disease due to that agent. Passive antibody administration is the only means of providing immediate immunity to susceptible persons and immunity of any measurable kind for highly immunocompromised patients.

Passive antibody therapy has a history going back to the 1890 s and was the only means of treating certain infectious diseases prior to the development of antimicrobial therapy in the 1940s (3, 4). Experience from prior outbreaks with other coronaviruses, such as SARS-CoV-1 shows that such convalescent plasma contains neutralizing antibodies to the relevant virus (5). In the case of SARS-CoV-2, the anticipated mechanism of action by which passive antibody therapy would mediate protection is viral neutralization. However, other mechanisms may be possible, such as antibody dependent cellular cytotoxicity and/or phagocytosis. Convalescent serum was also used in the 2013 African Ebola epidemic. A small non-randomized study in Sierra Leone revealed a significant increase in survival for those treated with convalescent whole blood relative to those who received standard treatment (6).

The only antibody type that was available for immediate use at the peak of the covid-19 epidemic was that found in human convalescent plasma. As more individuals contracted covid-19 and recovered, the number of potential donors was expected to increase.

\section{2 | MATERIALS AND METHODS}

\section{Study design}

This was an open label, single centre, two arm, prospective, randomised, controlled exploratory phase 2 study conducted at a COVID-19 designated center in Mumbai city, one of the biggest covid-19 disease epicentres in India. 20 adults ( $\geq 18$ years) admitted to hospital (screened from 15 June to 27 July 2020) with confirmed moderate covid-19 (partial pressure of oxygen in arterial blood/ fraction of inspired oxygen $(\mathrm{PaO} 2 / \mathrm{FiO} 2)$ ratio between 200 $\mathrm{mm} \mathrm{Hg}$ and $300 \mathrm{~mm} \mathrm{Hg}$ or a respiratory rate of more than $24 /$ min with oxygen saturation $93 \%$ or less on room air): 10 subjects were assigned to convalescent plasma with standard treatment (test arm) and 10 subjects to standard treatment only (control arm). Subjects in the test arm received either single or 2 doses of convalescent plasma 24 hours apart based on their clinical condition as per investigator's discretion.

\section{Ethics}

The study was commenced at the site only after a written approval was obtained from the Wockhardt Hospital Institutional Ethics Committee and Central Drugs Standard Control Organisation (CDSCO),

Supplementary information The online version of this article (https://doi.org/10.15520/jcmro.v4i05.41 9) contains supplementary material, which is available to authorized users.

\section{Corresponding Author: M. Shah}

Wockhardt's Hospital, near Police Station, 1877, Doctor Anandrao Nair Marg, Near Agripada, Mumbai Central, Mumbai, Maharashtra, India - 400011

Email: Manish.Shah@wockhardt.com 
which is India's national regulatory body for pharmaceuticals and medical devices.

Trial registration: Clinical Trial Registry of India CTRI/2020/05/025299.

\section{Subjects}

Subjects aged at least 18 years who had confirmed covid-19 based on a positive reverse transcriptase polymerase chain reaction (RT-PCR) result for SARSCoV-2 and had been admitted to the participating hospital were screened for eligibility. Inclusion criteria were moderate illness with either a $\mathrm{PaO} 2 / \mathrm{FiO} 2$ ratio between $200 \mathrm{~mm} \mathrm{Hg}$ and $300 \mathrm{~mm}$ $\mathrm{Hg}$ or a respiratory rate of more than $24 / \mathrm{min}$ with oxygen saturation ( $\mathrm{SpO} 2) 93 \%$ or less on room air, and availability of a matched donor for convalescent plasma at the point of enrolment. Pregnant and lactating women, patients with known hypersensitivity to blood products, recipients of immunoglobulin in the past 30 days, patients with conditions precluding infusion of blood products, participants in any other clinical trials, and critically ill patients with $\mathrm{PaO} 2 /$ FiO2 $<200 \mathrm{~mm} \mathrm{Hg}$ or shock (requiring vasopressors to maintain a mean arterial pressure (MAP) of $\geq 65$ mm Hg or MAP of $<65 \mathrm{~mm} \mathrm{Hg}$ ) were excluded.

Eligible donors were men or nulliparous women who were aged between 18 and 65 years, weighed more than $50 \mathrm{~kg}$, had received a diagnosis of covid-19 confirmed by a RT-PCR test result, and had experienced symptoms of covid-19 with at least fever and cough. Additionally, the symptoms had completely resolved for 28 consecutive days before donation or a period of 14 days before donation with two negative RT-PCR test results for SARS-CoV-2 from nasopharyngeal swabs collected 24 hours apart. All routine screening tests, including ABO blood grouping; Rhesus phenotype; complete blood counts; screening for HIV, hepatitis B or C virus, syphilis, and malaria; and total serum protein were conducted according to the Drugs and Cosmetics ( $2^{\text {nd }}$ amendment) Rules, 2020.

All participants or their family members or legally authorised representatives were provided with information about the trial in a language with which they were familiar, and written informed consent was obtained before starting any study specific procedure.

\section{Procedures}

Patients enrolled in the control arm received best standard treatment for covid-19 in keeping with the institutional protocol, which was dictated by the best available evidence at the time and guidelines for the management of covid-19 issued by health authorities of the Indian government. The range of treatment protocols followed by the participating clinical site for the management of patients with covid-19 included antivirals (hydroxychloroquine, remdesivir, oseltamivir), broad spectrum antibiotics, immunomodulators (steroids, tocilizumab, pirfenidone), and supportive management (oxygen through a nasal cannula, face mask, etc.). The decision to transfer to the intensive care unit depended on the policies of the hospital.

Participants in the test arm received either a single dose of $200 \mathrm{~mL}$ of convalescent plasma, or 2 doses transfused 24 hours apart (depending on the participant's clinical condition as per investigator's discretion), in addition to the best standard treatment. The dose of convalescent plasma was transfused after the subject was allocated to the test arm. Convalescent plasma was collected from patients who had recovered from covid-19 by centrifugal separation using the apheresis equipment available at the assigned blood bank after obtaining written informed consent from the donors.

All participants underwent clinical examination and a range of laboratory investigations, including arterial blood gas analysis, complete blood count, renal and hepatic function tests, and a coagulation profile on the day of enrolment (day 0 ) and subsequently on days $1,3,5,7$, and 14. Chest imaging was carried out on days 0,14 , and 28 . Serum for antibody titre assays was collected on days $0,1,3,7$ and 14; these samples were stored at $-80^{\circ} \mathrm{C}$ until further analysis at Wockhardt hospital research \& development center, Mumbai, India. RT-PCR test using nasopharyngeal swabs was repeated on days $0,1,3,7$ and 14. All participants visited the hospital on day 28 to assess their health status.

Enzyme Immuno assay was used for the quantification of IgG antibodies. Two quantification kits were used [both approved by Indian Council of Medical Research (ICMR)]; GENLISATM Human Anti- 
Convalescent Plasma to Limit Coronavirus Associated Complications: A Proof of Concept Phase-2 Clinical Study at a designated COVID-19 hospital in Mumbai city

SARS-CoV-2 (Covid-19) IgG spike protein Quantitative Titration ELISA (Cat No.KBVH015-8) and GENLISA $^{\mathrm{TM}}$ Human Anti-SARS-CoV-2 (Covid19) IgG Antibody to nucleoprotein Quantitative ELISA (Cat No\# KBVH015-9). Antibody titers were assayed as per the manufacturer's instructions. All samples and standards were run in duplicate. The mean absorbance for each set of duplicate standards and samples was determined. Standard concentration $(9 \mathrm{ng} / \mathrm{ml})$ was plotted against the absorbance at 450 $\mathrm{nm}$. Samples absorbance values were calculated by comparing to the standards.

\section{Outcomes}

The primary outcome of the study was avoidance of progression to severe disease $(\mathrm{PaO} 2 / \mathrm{FiO} 2$ ratio $<100$ $\mathrm{mm} \mathrm{Hg}$ ) any time within 28 days of enrolment.

The secondary outcomes were time to symptom resolution of fever, shortness of breath, or cough; total duration of respiratory support (invasive or noninvasive ventilation) during hospital admission, and post-enrolment duration of respiratory support until day 28 or discharge; radiological improvement; conversion to a negative result for RT-PCR test on days $1,3,5,7$, and 14; RT-PCR test was repeated at Day 28 only for those subjects whose test was positive at Day 14; hospital mortality \& length of stay (LOS); intensive care unit (ICU) mortality and LOS; and 28 days mortality.

Safety outcomes were rapid deterioration of respiratory or clinical status on transfusion of SARS-CoV2 convalescent plasma and cumulative incidence of adverse events (AEs) and serious adverse event during the study period. Relatedness of AE with the trial was assessed according to published definitions.

\section{3 | STATISTICAL ANALYSIS}

\section{Primary Efficacy Endpoint:}

The primary efficacy endpoint was computed as the proportion of subjects who avoid progression to severe ARDS $(\mathrm{PaO} 2 / \mathrm{FiO} 2$ ratio < 100) within Day 28 of start of convalescent plasma therapy. This proportion was assessed in each treatment arm and compared between treatments using the Fisher's exact test at the $5 \%$ level of significance.

\section{Secondary Efficacy Endpoints:}

The time to symptom resolution of fever, shortness of breath and cough was presented using KaplanMeier plots and analysed using the log-rank test. The duration of required respiratory support was computed for both invasive and non-invasive support methods and analysed in a similar manner. The change from baseline in RNA levels of SARS-CoV2 was planned to be plotted over time until 14 days after transfusion and compared between treatment arms using an appropriate ANCOVA model. The change from baseline in SARS-COV-2 neutralizing antibody titres was analysed in a similar manner.

The median LOS in ICU, in the hospital and the median number of ventilator-free days was planned to be computed and compared between treatment arms using an appropriate non-parametric test.

All-cause mortality, hospital mortality and ICU mortality were summarized by treatment arm.

\section{Safety Endpoints:}

The key safety endpoint to be assessed was rapid deterioration of respiratory or clinical status including transfusion related reactions after transfusion of convalescent plasma therapy and cumulative incidence of adverse events and serious adverse events during the study duration.

\section{Analysis population:}

The various analysis populations in the study were defined as below:

Modified intent to treat (mITT) population: The mITT population was used for the analysis of all efficacy endpoints. All subjects who were administered at least one unit of convalescent plasma transfusion and/or standard treatment and provided a baseline and at least one treatment efficacy measurement.

Per-protocol (PP) Population: All subjects who completed the study as per the protocol with no major protocol deviations.

Safety population: All subjects who received at least one unit of convalescent plasma transfusion and/or standard treatment were used for safety analysis. 


\section{4 | RESULTS}

\section{Subject disposition and analysis sets}

A total of 20 subjects were allocated in an alternate sequential manner in 1:1 ratio; of these 10 subjects were allocated into test arm and received standard treatment plus convalescent plasma and 10 subjects were allocated into the control arm and received standard treatment only. A total of 19 subjects completed the study and 1 subject in the control arm was discontinued from the study due to other reason [subject was contacted on Day-28 but he was out of station hence did not report for Day-28 visit. He had no complaint regarding his health]Table 1.

\section{Subject demographic and clinical characteristics}

All subjects were of the Indian race. Among all subjects enrolled in the study (mITT), 14 were male $(70 \%)$ and 6 were female $(30 \%)$. The median age of subjects was 56.50 years (range: $27-70$ years of age). The median body mass index was $26.23 \mathrm{~kg} / \mathrm{m}^{2}$ (range: $19.86-32.09 \mathrm{~kg} / \mathrm{m}^{2}$ ). None of the subjects reported travel history in past 21 days from screening day. 4 subjects reported positive contact with Covid19 positive in past 21 days from screening day Table 2 .

Baseline personal and clinical characteristics, available for all 20 participants, were similar across the trial arms. Patient management across the trial arms was similar except for convalescent plasma treatment.

All subjects (20 subjects [100\%]) received at least one prior or concomitant medication. The most common classes of prior or concomitant medications (i.e., reported in $\geq 10$ [50.0\%] subjects) included: Vitamins (20 subjects [100.0\%]); Corticosteroids for systemic use (20 subjects [100.0\%]); Drugs for acid related disorders (20 subjects [100.0\%]); Antithrombotic agents (18 subjects [90.0\%]); Analgesics (16 subjects [80.0\%]); Antibacterial for systemic use (16 subjects [80.0\%]); Drugs used in diabetes (15 subjects [75.0\%]); Drugs for Constipation (13 subjects [65.0\%]); Antivirals for systemic use (12 subjects [60.0\%]); Immunosuppressants (10 subjects $[50.0 \%])$.

\section{5 | CLINICAL OUTCOMES}

\section{Efficacy}

None of the subject (mITT population) in the test arm progressed to severe ARDS $\left(\mathrm{PaO} 2 / \mathrm{FiO}_{2}<100\right)$; only 1 subject in the control arm progressed to severe ARDS $(\mathrm{PaO} 2 / \mathrm{FiO} 2$ ratio $<100)$ - Table 3.

Median time to resolution of fever in mITT population was 2 days less in the test arm as compared to the control arm (13.00 vs 15.00 days, respectively). Median time to resolution of shortness of breath in mITT population was 3 days less in the test arm as compared to the control arm (13.00 vs. 16.00 days, respectively). Median time to resolution of cough in mITT population was 3 days less in the test arm as compared to the control arm (18.00 vs. 21.00 days, respectively) - Table 4 Similar results were observed in the PP population.

Post baseline, none of the subjects in the safety population showed abnormal clinically significant CT scan changes at the end of study (EOS) visit except one subject in the control arm.

Median duration of RT-PCR test turning negative in the mITT population was 9.5 days less in the test arm as compared to control arm (9.0 vs 18.50 days, respectively). Similar results were observed in the PP population. Maximum change in the proportion of subjects (mITT population) of RT-PCR test turning negative was observed on Day 3 and Day 14, similar results were observed in the PP population.

Median duration (mITT population) of non-invasive respiratory support was similar in the test and control arms (8.00 days); however, median duration of noninvasive respiratory support in the PP population was 1.5 days less in the test arm as compared to control arm (6.50 vs. 8 days, respectively).

No subject required mechanical (invasive) ventilation in the study.

There was no ICU mortality or hospital mortality observed during the study.

Median duration of LOS in ICU in the mITT population was 8 days and 7 days in the test and control arm; i.e, one day less in control arm as compared to the test arm, respectively and the median LOS in hospital was 12 days in both the arms. Similar results 
Convalescent Plasma to Limit Coronavirus Associated Complications: A Proof of Concept Phase-2 Clinical Study at a designated COVID-19 hospital in Mumbai city

TABLE 1: Subject Disposition (Modified ITTPopulation)

\begin{tabular}{|c|c|c|c|}
\hline & $\begin{array}{l}\text { Test arm } \\
(\mathrm{N}=10)\end{array}$ & $\begin{array}{l}\text { Control arm } \\
(\mathrm{N}=10)\end{array}$ & $\begin{array}{l}\text { Overall } \\
(\mathrm{N}=20)\end{array}$ \\
\hline Number of subjects & $\mathrm{n}(\%)$ & $\mathrm{n}(\%)$ & $\mathrm{n}(\%)$ \\
\hline Randomised & 10 & 10 & 20 \\
\hline Completed & $10(100.0)$ & $9(90.0)$ & $19(95.0)$ \\
\hline Discontinued & $0(0.0)$ & $1(10.0)$ & $1(5.0)$ \\
\hline $\begin{array}{l}\text { Reasons for Discontinuation } \\
\text { Other }\end{array}$ & $0(0.0)$ & $1(10.0)$ & $1(5.0)$ \\
\hline Safety population & $10(100.0)$ & $10(100.0)$ & 20 100.0) \\
\hline Per protocol population & $8(80.0)$ & $7(70.0)$ & $15(75.0)$ \\
\hline Modified intent treat population & $10(100.0)$ & $10(100.0)$ & 20 100.0) \\
\hline \multirow{2}{*}{\multicolumn{4}{|c|}{$\begin{array}{l}\text { Source: Table } 14.1 .2 \text { of Clinical Study Report Version } 1.0 \text { dated } \\
\text { 21/Dec/2020 } \\
\text { Note: Percentages (\%) are based upon number of subjects randomized } \\
\text { in respective treatment arms. }\end{array}$}} \\
\hline & & & \\
\hline \multicolumn{4}{|c|}{$\begin{array}{l}\text { Note: Modified Intent-to-treat (mITT) Population: All subjects who re- } \\
\text { ceived at least one dose of study medication and have at least one post } \\
\text { baseline efficacy assessment. }\end{array}$} \\
\hline \multicolumn{4}{|c|}{$\begin{array}{l}\text { Note: Per-protocol (PP) Population: All subjects who completed the } \\
\text { study as per the protocol with no major protocol deviations. }\end{array}$} \\
\hline \multicolumn{4}{|c|}{$\begin{array}{l}\text { Note: Safety Population: All subjects who received at least one dose of } \\
\text { study medication. }\end{array}$} \\
\hline \multicolumn{4}{|c|}{ Reference Listing: Listing 16.2.1.2, 16.2.1.3 and 16.2.2. } \\
\hline \multicolumn{4}{|c|}{$\begin{array}{l}\text { Note: "Other" reason: Subject was contacted on Day- } 28 \text { but he was out } \\
\text { of station hence did not report for Day- } 28 \text { visit. He has no Complaint } \\
\text { regarding his health }\end{array}$} \\
\hline
\end{tabular}

were observed in the PP population for both LOS in ICU and hospital.

There was no significant difference observed between the 2 groups for Anti-SARS-CoV2 antibody titer values. Both groups showed increasing titers from baseline to EOS, however the median change from baseline (test arm: 16.15 AU/ml; control arm: 45.52 AU/ml) anti-spike protein immunoglobulin $\mathrm{G}$ (IgG) concentration was observed to be more in the test arm as compared to the control arm at EOS (test arm: 159.35 AU/ml; control arm: $149.70 \mathrm{AU} / \mathrm{ml}$ ). Further studies on the intensity of disease and donor antibody titers combined with neutralizing antibody titers may help assess the efficacy of convalescent plasma in various disease stages.

\section{Safety}

There were no deaths or SAEs reported during the study. A total of 17 subjects (85.0\%) reported 24 events: 9 subjects in the test arm reported 10 events and 8 subjects in the control arm reported 14 events. The three most frequently affected SOCs were Gastrointestinal Disorders ( 8 subjects, Respiratory, Thoracic and Mediastinal Disorders ( 7 subjects, Vascular Disorders (3 subjects) with most frequent reported AEs by preferred term (PT) were ARDS (7 subjects) and constipation ( 5 subjects). One subject in the test arm experienced AE of bradycardia (non-serious) which was assessed by the investigator as related to the study drug. Severe event of ARDS was reported in 1 subject in the control arm Table 5. 
TABLE 2: Demographic and Baseline Characteristics(Modified ITT Population)

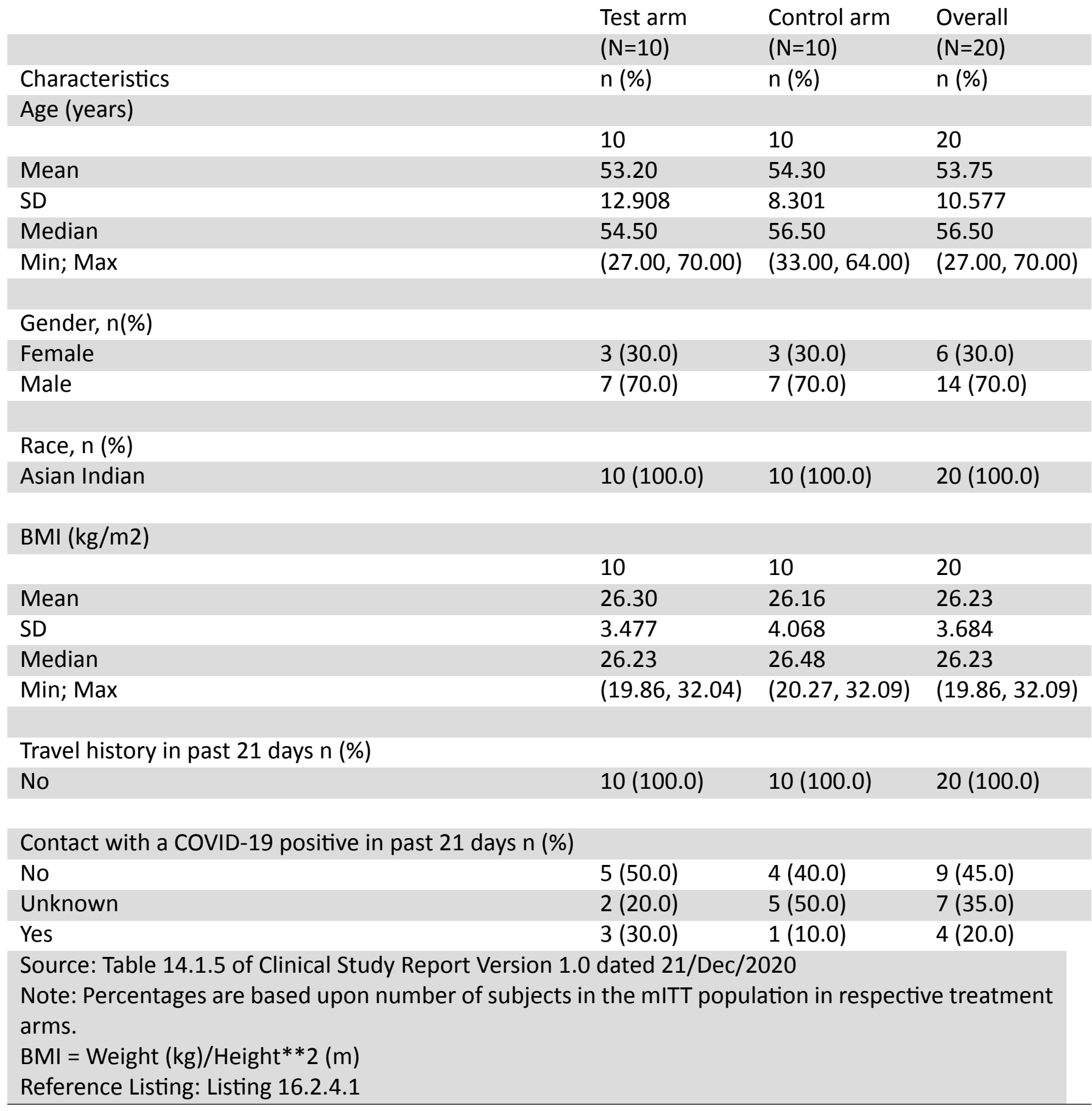

\section{6 | DISCUSSION}

With few treatment options available to manage coronavirus disease 2019 (covid-19), the disease presented a unique set of challenges for healthcare providers globally. In addition to using non-drug interventions, health systems had devised strategies to manage covid-19 using repurposed drugs and revisit- ing older strategies, such as convalescent plasma. In the past, convalescent plasma was used as a passive immunisation strategy to treat viral diseases, raising expectations that potentially it could be used to treat severe acute respiratory syndrome coronavirus 2 (SARS-CoV-2), the virus responsible for covid19 and a disease with no proven, effective interventions $(7,8)$. 
Convalescent Plasma to Limit Coronavirus Associated Complications: A Proof of Concept Phase-2 Clinical Study at a designated COVID-19 hospital in Mumbai city

TABLE 3: Proportion of Subjects who Avoid Progression toSevere ARDS (PaO2/ FiO2 Ratio < 100) Within Day 28 of Randomisation (mITTPopulation)

\begin{tabular}{llll} 
& Test arm & $\begin{array}{l}\text { Control } \\
\text { arm }\end{array}$ & $\begin{array}{l}P \\
\text { value }\end{array}$ \\
Category of PaO2/FiO2 ratio & $\mathrm{n} / \mathrm{N}(\%)$ & $\mathrm{n} / \mathrm{N}(\%)$ & \\
Proportion of subjects who avoid progression to severe ARDS & $10 / 10$ & $9 / 10$ & $>0.999$ \\
(PaO2/FiO2 ratio $<100)$ & $(100.0)$ & $(90.0)$ & \\
$\begin{array}{l}\text { Source: Table 14.2.1 of Clinical Study Report Version 1.0 dated 21/Dec/2020 } \\
\text { Reference Listings: Listing 16.2.6.1est }\end{array}$ & & \\
\hline
\end{tabular}

TABLE 4: The Time to Symptom Resolution of Fever,Shortness of Breath, and Cough (mITT Population)

\begin{tabular}{|c|c|c|}
\hline & Test arm & Control arm \\
\hline Symptoms & $(\mathrm{N}=10)$ & $(\mathrm{N}=10)$ \\
\hline \multicolumn{3}{|l|}{ Fever } \\
\hline & 5 & 5 \\
\hline Mean & 13.20 & 15.20 \\
\hline SD & 0.84 & 5.26 \\
\hline Median & 13.00 & 15.00 \\
\hline Min; Max & $(12,14)$ & $(8,22)$ \\
\hline KM estimate of the median time $(95 \% \mathrm{Cl})$ & $13.0(12.00,14.00)$ & $15.0(8.00,22.00)$ \\
\hline P-value & & 0.1635 \\
\hline \multicolumn{3}{|l|}{ Shortness of breath } \\
\hline & 10 & 10 \\
\hline Mean & 17.90 & 17.60 \\
\hline SD & 10.91 & 6.67 \\
\hline Median & 13.00 & 16.00 \\
\hline Min; Max & $(6,43)$ & $(11,33)$ \\
\hline KM estimate of the median time $(95 \% \mathrm{Cl})$ & $13.0(6.00,23.00)$ & $16.0(11.00,21.00)$ \\
\hline P-value & & 0.7613 \\
\hline \multicolumn{3}{|l|}{ Cough } \\
\hline & 5 & 7 \\
\hline Mean & 17.00 & 21.57 \\
\hline SD & 6.16 & 5.50 \\
\hline Median & 18.00 & 21.00 \\
\hline Min; Max & $(8,23)$ & $(16,33)$ \\
\hline KM estimate of the median time $(95 \% \mathrm{Cl})$ & $18.0(8.00,23.00)$ & $21.0(16.00,22.00)$ \\
\hline P-value & & 0.5202 \\
\hline \multicolumn{3}{|c|}{$\begin{array}{l}\text { Source: Table } 14.2 .2 \text { of Clinical Study Report Version } 1.0 \text { dated 21/Dec/2020 } \\
\text { Reference Listings: Listing 16.2.6.2 }\end{array}$} \\
\hline
\end{tabular}




\section{GURRENT MEDICAL RESEARCH AND OPINION}

TABLE 5: Incidenceof Adverse Events Irrespective of Causality (Safety Population)

\begin{tabular}{|c|c|c|c|}
\hline & Test arm & Control arm & Overall \\
\hline System Organ Class (SOC) & $(\mathrm{N}=10)$ & $(\mathrm{N}=10)$ & $(\mathrm{N}=20)$ \\
\hline Preferred Term (PT) & $\mathrm{n}(\%)$ & $\mathrm{n}(\%)$ & $\mathrm{n}(\%)$ \\
\hline Number of subjects with at least one Adverse Event & $9(90.0)$ & $8(80.0)$ & $17(85.0)$ \\
\hline Blood and lymphatic system disorders & $0(0.0)$ & $1(10.0)$ & $1(5.0)$ \\
\hline Anemia & $0(0.0)$ & $1(10.0)$ & $1(5.0)$ \\
\hline Cardiac disorders & $1(10.0)$ & $0(0.0)$ & $1(5.0)$ \\
\hline Bradycardia & $1(10.0)$ & $0(0.0)$ & $1(5.0)$ \\
\hline Gastrointestinal disorders & $4(40.0)$ & $4(40.0)$ & $8(40.0)$ \\
\hline Constipation & $3(30.0)$ & $2(20.0)$ & $5(25.0)$ \\
\hline Haematochezia & $0(0.0)$ & $1(10.0)$ & $1(5.0)$ \\
\hline Mouth ulceration & $1(10.0)$ & $0(0.0)$ & $1(5.0)$ \\
\hline Stomatitis & $0(0.0)$ & $1(10.0)$ & $1(5.0)$ \\
\hline Infections and infestations & $1(10.0)$ & $1(10.0)$ & $2(10.0)$ \\
\hline Fungal skin infection & $1(10.0)$ & $0(0.0)$ & $1(5.0)$ \\
\hline Oral candidiasis & $0(0.0)$ & $1(10.0)$ & $1(5.0)$ \\
\hline Metabolism and nutrition disorders & $0(0.0)$ & $1(10.0)$ & $1(5.0)$ \\
\hline Hyperglycaemia & $0(0.0)$ & $1(10.0)$ & $1(5.0)$ \\
\hline Respiratory, thoracic and mediastinal disorders & $2(20.0)$ & $5(50.0)$ & $7(35.0)$ \\
\hline Acute respiratory distress syndrome & $2(20.0)$ & $5(50.0)$ & $7(35.0)$ \\
\hline Vascular disorders & $2(20.0)$ & $1(10.0)$ & $3(15.0)$ \\
\hline Haematoma & $0(0.0)$ & $1(10.0)$ & $1(5.0)$ \\
\hline Hypertension & $1(10.0)$ & $0(0.0)$ & $1(5.0)$ \\
\hline Hypotension & $1(10.0)$ & $0(0.0)$ & $1(5.0)$ \\
\hline \multicolumn{4}{|c|}{ Source: Table 14.3.2 of Clinical Study Report Version 1.0 dated 21/Dec/2020 } \\
\hline \multicolumn{4}{|c|}{ Note: Adverse events were coded using MedDRA dictionary (version: 23.0). } \\
\hline \multicolumn{4}{|c|}{$\begin{array}{l}\text { Note: Subjects having multiple AEs within same System Organ Class (SOC) were counted } \\
\text { only once in respective system organ class under any event. }\end{array}$} \\
\hline \multicolumn{4}{|c|}{$\begin{array}{l}\text { Note: Subjects having multiple AEs with same Preferred Term (PT) were counted only } \\
\text { once within the respective PT. }\end{array}$} \\
\hline \multirow{2}{*}{\multicolumn{4}{|c|}{$\begin{array}{l}\text { Note: SOC has been sorted alphabetically and PTs have been sorted within each SOC } \\
\text { alphabetically. } \\
\text { Note: Percentages are based upon number of subjects in the safety population in } \\
\text { respective treatment arms }\end{array}$}} \\
\hline & & & \\
\hline Reference Listings: Listing 16.2.7.1 & & & \\
\hline
\end{tabular}


Since the publication of the first case series from China, multiple observational studies have been published, some on preprint servers, reporting the association between convalescent plasma and reduced mortality, hospital stay, and viral load in patients with covid-19 $(8-10)(11,12)$. The uncertainty on both the safety and effectiveness of convalescent plasma as a treatment option in patients admitted to hospital with covid-19 was reflected in all these published literature. Meanwhile, convalescent plasma treatment had received emergency regulatory approval for use in patients in few countries. This had resulted in its widespread adoption in real world clinical practice, where it was being used to treat patients with a wide spectrum of covid-19 severity. A large open label, parallel arm, phase II, multicentre, randomised controlled trial called as PLACID trial was conducted by Indian Council of Medical Research (ICMR). It was conducted in 39 tertiary care hospitals across India comprising of 464 recruited subjects. This trial result also showed that although the use of convalescent plasma seemed to improve resolution of shortness of breath and fatigue in patients with moderate covid-19 and led to higher negative conversion of SARS-CoV-2 RNA on day 7 postenrolment, this did not translate into a reduction in 28 day mortality or progression to severe disease (13).

This study was based on the PLACID trial protocol and ICMR guidelines. Study result showed that treatment with convalescent plasma was associated with lesser median time in the resolution of fever, shortness of breath and cough. At baseline, all subjects (safety population) showed abnormal clinically significant CT scan changes (e.g. ground glass opacities, consolidation, interstitial abnormalities, etc.); however only 1 subject in control arm continued to show abnormal clinically significant changes at the end of study (EOS); while in the remaining 19 subjects there were no clinically significant CT changes indicating recovery of the disease.

Subjects in the test arm took lesser number of median days for RT-PCR test to turn negative as compared to those in the control arm and the maximum change in the proportion of subjects of RT-PCR test turning negative was observed on Day 3 and Day 14.

The median duration of non-invasive respiratory support was similar in both the test and control arms and no subjects required mechanical (invasive) ventilation in the study.

There was no ICU mortality or hospital mortality observed during the study. Median duration of the LOS in both ICU as well as hospital was similar in both the test and control arm.

There was no significant difference observed between the 2 groups for Anti-SARS-CoV2 antibody titer values. Both groups showed increasing titers from baseline to EOS, however the median change from baseline antibody titer was observed to be more in the test arm as compared to the control arm.

\section{7 | CONCLUSION}

This was an exploratory POC study to explore the effectiveness of convalescent plasma in covid-19 subjects and sample size was not large enough to detect a statistically significant difference; however subjects who received convalescent plasma on top of standard treatment in test arm of this study showed better outcomes in few of the efficacy parameters as compared to subjects in control arm who received standard treatment only. Subjects in test arm showed earlier resolution of symptoms of fever, shortness of breath and cough and the mean duration for RTPCR test turning negative was better in the test arm. One subject in the control arm progressed to severe ARDS, while none in test arm progressed to severe ARDS. There was no serious adverse reaction observed with the transfusion of convalescent plasma in the study. This study result could help to facilitate planning of a confirmatory well-designed randomised Phase-III clinical trial.

\section{Acknowledgements}

We thank Dr. Maulek Desai, study participants, the principal investigator, other sub-investigators (Dr. Prashant Zore and Dr. Gurudas Bundpal), nurses, study coordinator and blood bank personnel (Dr. Kishore Jha) who made this study possible.

\section{Funding}

This study was funded by Wockhardt Hospital, which is a subsidiary of Wockhardt Ltd. 


\section{CURRENT MEDICAL RESEARCH AND OPINION}

\section{REFERENCES}

1. Patel AK, Patel KK, Patel KR, Shah S, Dileep $\mathrm{P}$. Time trends in the epidemiology of microbial infections at a tertiary care center in west India over last 5 years. J Assoc Physicians India 2010; 58 (Suppl): 37-40;.

2. Ministry of Health and Family Welfare, Government of India. COVID-19 India dashboard. Retrieved from, https://www.mohfw.gov.in.;

3. Casadevall A, Scharff MD. Return to the past: the case for antibody-based therapies in infectious diseases. Clinical infectious diseases. 1995;21(1):150-161.;.

4. Casadevall A, Dadachova E, Pirofski L. Passive antibody therapy for infectious diseases. Nature Reviews Microbiology. 2004;2(9):695-703.;.

5. Zhang J-S, Chen J-T, Liu Y-X, Zhang Z-S, Gao $\mathrm{H}$, Liu $\mathrm{Y}$, et al. A serological survey on neutralizing antibody titer of SARS convalescent sera. Journal of medical virology. 2005;77(2):147150.;.

6. Sahr F, Ansumana R, Massaquoi T, Idriss B, Sesay F, Lamin J, et al. Evaluation of convalescent whole blood for treating Ebola Virus Disease in Freetown, Sierra Leone. Journal of Infection. 2017;74(3):302-309.;.

7. Casadevall A, Joyner MJ, Pirofski LA. SARS-CoV-2 viral load and antibody responses: the case for convalescent plasma therapy. J Clin Invest 2020;130:51124.doi:10.1172/JCI139760.;.

8. Shen C, Wang Z, Zhao F, et al. Treatment of 5 Critically Ill Patients With COVID-19 With Convalescent Plasma. JAMA 2020;323:1582-9. doi:10.1001/jama.2020.4783.;.
9. Abolghasemi H, Eshghi P, Cheraghali AM, et al. Clinical efficacy of convalescent plasma for treatment of COVID-19 infections: Results of a multicenter clinical study. Transfus Apher Sci 2020;102875.doi:10.1016/j.transci.2020.102875.;.

10. Hegerova L, Gooley TA, Sweerus KA, et al. Use of convalescent plasma in hospitalized patients with COVID-19: case series. Blood 2020;136:759-62. doi:10.1182/blood.2020006964.;.

11. Xia X, Li K, Wu L, et al. Improved clinical symptoms and mortality among patients with severe or critical COVID-19 after convalescent plasma transfusion. Blood 2020;136:755-9. doi:10.1182/blood.2020007079;

12. Joyner MJ, Bruno KA, Klassen SA, et al. Safety Update: COVID-19 Convalescent Plasma in 20 000 Hospitalized Patients. Mayo Clin Proc 2020 Jul 19; www.ncbi.nlm.nih.gov/pmc/articles/PM C7368917/Accessed on 25 Aug 2020.;.

13. Agarwal A, Mukherjee A, Kumar G, et al. Convalescent plasma in the management of moderate covid-19 in adults in India: open label phase II multicentre randomised controlled trial (PLACID Trial). BMJ 2020; 371:m3939.;.

How to cite this article: Pardiwalla B., Yadav A., Bhatia A., Toraskar K., Sharma V., Shah M., Gutte R., Kumbhar D., Gohel D., Narayan P., Savla H., Nagda N., Bopardikar A. Convalescent Plasma to Limit Coronavirus Associated Complications: A Proof of Concept Phase-2 Clinical Study at a designated COVID-19 hospital in Mumbai city. Journal of Current Medical Research and Opinion. 2021;940-949. https://doi.or g/10.15520/jcmro.v4i05.419 